Jill McTavish (jmctavi2@uwo.ca) and Alexandre Fortier (afortie@uwo.ca)

The University of Western Ontario, London, Ontario, Canada

\title{
A Domain-Analytic Perspective on Sexual Health in LCSH and RVM
}

\begin{abstract}
This paper analyses and compares the treatment of sexual health in Library of Congress Subject Headings (LCSH) and Répertoire de vedettes-matière de l'Université Laval (RVM) using three of Bowker and Star's (1999) infrastructural inversion techniques: practical politics, convergence, and resistance. Our findings reveal that neither LCSH nor RVM offer a holistic representation of sexual health (practical politics), that LCSH's topical representation of sexual health limits access to relevant material (convergence), and that the enhancement of LCSH through user-added content could improve but not replace these systems (resistance).
\end{abstract}

\section{Background}

Information organization theory no longer assumes that systems for organizing information are objective or neutral; instead, all systems are seen to contain bias and limits (Olson and Ward 1997; Mai 2010). As Feinberg discusses, such a shift in theory "might benefit from an increasing focus on design, with a corresponding de-emphasis on discovery in the mode of science" (2007, n.p.). The design of information organization systems is "not merely a technical task; it is a task that involves making ontological statements about the world and the relations among entities in the world” (Mai 2010, 635). The ontological decisions informing system design are unfortunately often "buried in archives (when records are kept at all) or built into software or the sizes and compositions of things" (Bowker and Star 1999, 45). A task of information organization theory might therefore be to unbury the ontological decisions informing these systems. As Mai $(2010,639)$ argues about classification systems, in order for these systems to be trusted, designers and editors "must embrace the principle of transparency and explain their decisions and show the conceptual and philosophical foundations for their systems". In this paper, we suggest that Bowker and Star's (1999) six infrastructural inversion techniquesubiquity, materiality, indeterminancy, practical politics, convergence and resistance-can aid us in the task of making visible the ontological decisions informing knowledge organization systems. These strategies attend to the moral, scientific, and aesthetic implications of knowledge organization systems. They act as a sort of "gestalt switch" by allowing us to see the invisible, intricate "technologies and arrangements that, by design and by habit, tend to fade into the woodwork” (Bowker and Star 1999, 34).

Bowker and Star's (1999) project is pragmatic in nature, as they investigate “empirically how people have designed and used classification systems" and "how political and semantic conflicts are managed over long periods of time and at large levels of scale” (Bowker and Star 1999, p. 53). A pragmatic perspective is also central to the domain-analytic view, which is "mainly inspired by knowledge about the information structures in domains, by the sociology of knowledge and the theory of knowledge” (Hjørland and Albrechtsen 1995, 412). In this paper we follow McTavish, Neal, and Wathen's (forthcoming) suggestion that Bowker and Star's (1999) 
pragmatic strategies can be applied to other knowledge organization systems, such as alphabetic subject languages. We use three of Bowker and Star’s (1999) strategies_-practical politics, convergence, and resistance-in order to investigate two knowledge organization systems, Library of Congress Subject Headings (LCSH) and Répertoire de vedettes-matière (RVM), and their treatment of sexual health. Sexual health is a contentious topic where cultural differences in the two subject languages may emerge, thereby illustrating bias and limits.

\section{LCSH and RVM}

LCSH is a precoordinated indexing language that has been developed by the Library of Congress for more than a century. The language, which is built following the literary warrant approach, is strongly connected to the Library of Congress' collection. It is therefore not surprising that some of its content has a certain Americano-centric bias, a bias, however, that has lessened over the recent years. LCSH is developed around the principle of maintaining a certain stability (for "convenience of the user”), and this principle makes it harder to totally erase bias and limits in the tool, leaving historical oddities in the system. Some of these oddities in LCSH can be attributed to its long evolution without a set of guiding rules, and others to the fact that its syndetic structure mimic only loosely the guiding standards of controlled vocabularies (Chan 2005, Olson 2000).

RVM, which is developed by the Bibliothèque de l'Université Laval and supported by Library and Archives Canada, has been the national standard for French-language indexing in Canada since 1974. The tool, which originally debuted as a French translation of LCSH in the 1940s, is now considered to be a partial adaptation of the American subject heading list for the Francophone (and also Québécois) context. It is also a complete adaptation of the Canadian Subject Headings (a Canadian complement to LCSH) and gives many equivalent relationships to concepts from the Medical Subject Headings and the Art and Architecture Thesaurus. RVM, however, has a distinct character from its Anglophone counterparts, as indicated by its numerous "original” headings (more than 53,000 as of May 2011), i.e. headings without an English equivalent. These original heading depict realities that are ignored or incorrectly represented in the English tools, such as LCSH. Furthermore, the syndetic structure of translated headings does not have to follow those found in the original English tools, which results in entirely different realities in English and French. One patent example of different syndetic structures in LCSH and RVM is for the heading "sodomy”. In LCSH, this heading has "crimes without victims" and "sex crimes" as broader headings, while in RVM is has "relations sexuelles (sexual intercourse) (Dolbec 2006, Répertoire de vedettes-matières 2011).

\section{Definitions of Sexual Health}

The concept of sexual health has evolved over time and is historically and currently contentious (see, for example, the summary offered by Edwards and Coleman 2004). A working group for the World Health Organization $(1987,2)$, for instance, discussed how abstentionists and reformers have "used concepts about health to justify sexual norms, making use of their authority as experts". Further, the danger of defining sexual health can be that "it defines certain people's behaviour as 'healthy' and therefore defines as 'unhealthy' all those for a variety of individual reasons, feelings or values, do not fit within the accepted norm” (WHO 1987, 3). The need, however, for a comprehensive definition of sexual health has been cited for several reasons. Case definitions, or standardized criteria for determining whether a persona has a 
disease or health condition, are used for public health surveillance, clinical care, research, and service provision (see, for example, Chan and Donovan 2005). Operational health definitions are used to make strategic improvements in practice and systems and to improve health outcomes (Bialek et al. 2009; McDowell 2006). Comparable definitions across disciplines can also allow for transdisciplinary research, which can "see investigators move beyond the confines of the disciplines in which they are train” (Jordan 2009, 412).

A more recent meeting by the WHO $(2002,3)$ alludes to these very points, as they joined together to “discuss key concepts including definitions of sexual health and related concepts”, to "examine the specific barriers to the promotion of sexual health for adolescents and adults", and to "propose appropriate, effective strategies for promoting sexual health". While at this meeting the WHO $(2002,3)$ working group decided that delineating strategies for promoting sexual health "would not be useful given the very specific national and regional perspectives on how sexuality and thus sexual health can be addressed and promoted by the health sector", they did come to a consensus on a working definition of sexual health. In their final report, sexual health is defined as:

a state of physical, emotional, mental and social well-being related to sexuality; it is not merely the absence of disease, dysfunction or infirmity. Sexual health requires a positive and respectful approach to sexuality and sexual relationships, as well as the possibility of having pleasurable and safe sexual experiences, free of coercion, discrimination and violence. For sexual health to be attained and maintained, the sexual rights of all persons must be respects, protected, and fulfilled. (WHO 2002, 5) This definition relies on the WHO's $(1946,2)$ definition of health, as "a state of complete physical, mental and social well-being and not merely the absence of disease or infirmity”. It affirms the role of pleasure and respect in sexual relationships, the deleterious effect of violence on sexual relationships, and the importance of sexual rights. It also separates sexual health from a discussion of reproductive health or sexual development, which it instead relegates to its definition of sexuality. With this understanding of sexual health, we ask:

1. What design decisions inform LCSH and RVM categories and how do they shape the ability of these systems to represent the concept of sexual health (practical politics)?

2. How do LSCH and RVM headings and discourses of sexual health co-constitute one another (convergence)?

3. How have subject headings in LCSH and RVM evolved in comparison to current discourses on sexual health (resistance)?

\section{Methods}

We applied three of Bowker and Star's (1999) infrastructural inversion techniquespractical politics, convergence, and resistance, as described above-to the treatment of sexual health LCSH and RVM. Our data consists of subject headings related to sexual health and their syndetic structure contained in LCSH and RVM. We first briefly examined the history of bias in LCSH design and discuss how this bias has influenced the representation of sexual health (practical politics). To see how the concept of sexual health is framed depending on domain, we compared the constructions of sexual health in LCSH and RVM to the WHO's definition of sexual health and to sexual health discourses (convergence). To assess the state of written knowledge on sexual health, we sampled citations that referenced sexual health across four dates in order to represent four decades of sexual health information $(1980,1990,2000,2010)$. We sampled these four dates from within three different databases in order to reflect medical, psychological, and sociological representations of sexual health (Medline, PsycINFO, 
Sociological Abstracts) (see appendix I). We ran these citations (titles and abstracts) through word frequency analysis software to find the ten most frequent themes associated with sexual health (see appendix I). Finally, we examined the evolution of sexual health subject headings in LCSH and compared them to sexual health tags found in LibraryThing (http://www.librarything.com), a service that associates user-generated tags with an individual's personal library catalogue, in order to discuss the potential future reality of LCSH (resistance). Unfortunately, the low volume of documents currently tagged with "santé sexuelle" in the English and French version of Library Thing prevent us to make an adequate comparison with RVM in this respect.

\section{Results:}

\subsection{Practical Politics}

Practical politics refers to the design of infrastructure. Through the process of creating categories and standards, designers are "deciding what will be visible or invisible within the system” (Bowker and Star 1999, 44). Practical politics refers to both the content of categories and the structure of the overall system as these decisions determine "whose voice will be heard and when will enough date, of the right granularity, have been collected” (Bowker and Star 1999, 46). In this section we will briefly discuss the history of bias informing LCSH in particular and how this bias impacts the representation of sexual health in LCSH and RVM.

The bias in LCSH, in terms of lack of specificity in the language, outdated terminology, complicated syndetic structure, and inconsistent application of subdivisions, has been a point of contention in LIS since the late 1960s (see, for example, the bibliographic summary offered by Fisher 2005). While the limitations of LCSH are well-established, "few authors suggest that LCSH should be abandoned, indicating that while the subject heading lists is flawed, it is the best subject access tool available” (Fisher 2005, 75). The point of an investigation of practical politics is not to replace the system, although this may well occur, but to make a movement towards a more responsible representation of the social world that the information technology undergirds. We find this step necessary to make our information systems more transparent and therefore, as Mai (2010) suggests, more trustworthy.

The syndetic structure of sexual health in LCSH and RVM offer a distinct representation of sexual health. For example, the authorized heading in RVM is one of the rejected headings in LCSH: "sexual hygiene". In French, the term "hygiène" has the same definition as the English "hygiene": "The branch of knowledge that deals with the maintenance of health, esp. the conditions and practices conducive to it; the conditions and practices of a place or person in so far as they promote health.” (Shorter Oxford). In RVM, "santé” and "hygiène” are two authorized headings linked through an associative relationship, but this relationship is inexistent at the "sexual health" level ("santé sexuelle" is not even a rejected heading). Both the definitions of "santé" as commonly understood in French and the one given by RVM ("Sous cette vedette de sujet, on trouve les documents sur l'état de bien-être physique, mental et social complet ainsi que sur les soins pour l'acquérir et le préserver.") are more holistic than "hygiène” and are closer to WHO's definition of sexual health. In contrast, the narrower headings in RVM present a more complete portrait of sexual health, including contraception, sexual exercises and promiscuity and birth control. Sexual health is not linked to sexuality in either of the subject heading lists. 
Jill McTavish and Alexandre Fortier. 2011. A domain-analytic perspective on sexual health in LCSH and RVM. In Smiraglia, Richard P., ed. Proceedings from North American Symposium on Knowledge Organization, Vol. 3. Toronto, Canada, pp. 83-93.

\begin{tabular}{|l|l|l|l|}
\hline \multicolumn{2}{|l|}{} & LCSH & RVM \\
\hline \multicolumn{2}{|l|}{ Authorized heading } & Sexual health & Hygiène sexuelle \\
\hline Rejected headings & $\begin{array}{l}\text { Hygiene, Sexual [Former heading] } \\
\text { Hygiene, Social } \\
\text { Sex hygiene } \\
\text { Sexual hygiene } \\
\text { Social hygiene }\end{array}$ & Hygiène sociale \\
\hline $\begin{array}{l}\text { Hierarchical } \\
\text { relationships }\end{array}$ & Broader headings & Health & Hygiène \\
\cline { 3 - 4 } & Narrower headings & Safe sex in AIDS prevention & $\begin{array}{l}\text { Contraception } \\
\text { Exercices sexuels } \\
\text { Promiscuité } \\
\text { Régulation des naissances } \\
\text { Sexualité sans risque dans la } \\
\text { prévention du sida }\end{array}$ \\
\hline Associated headings & Maladies transmises sexuellement \\
\hline
\end{tabular}

\subsection{Convergence}

Convergence draws attention to how "information artifacts undergird social worlds, and [how] social worlds undergird these same information resources” (Bowker and Star 1999, 82). The point of convergence is to "pay more attention to the classification and standardization work...and so more deeply explore the terrain of the politics of science in action” (Bowker and Star 1999, 48). In order to discuss how LCHS and RVM converge with discourses on sexual health, we first briefly discuss some frequently occurring themes that are associated with sexual health, before going on to discuss the relationship between the subject headings and sexual health in LSCH and RVM.

In order to gauge the type of themes associated with sexual health, we sampled citations that referenced sexual health on four dates, 1980, 1990, 2000, and 2010, in order to reflect four decades of sexual health information. We sampled these four dates from within three different databases, Medline, PsycINFO, and Sociological Abstracts, in order to reflect medical, psychological, and sociological representations of sexual health (see appendix I). After running these citations (titles and abstracts) through word frequency analysis software, we found that the ten most frequent themes associated with sexual health were as follows: risk, sex or sexual intercourse, knowledge or information, HIV/AIDs, sexuality, STIs/STDs, reproduction, condoms, sexual education, and pregnancy (see appendix I). Many of the themes mentioned in the WHO's definition of sexual health, such as emotional, mental, and social well-being, pleasure, violence, and sexual rights, were not found in the ten the most frequently occurring themes. Each of these ten themes, however, are individually unsurprising as they reflect current health discourses, such as the influence of health promotion and the notion of risk.

The WHO $(1986,1)$ defines health promotion as the "process of enabling people to increase control over, and to improve their health". Sexual health promotion can entail "any activity that promotes positive sexual health, reduces the incidence of sexually transmitted infections (STIs) including HIV, reduces unintended pregnancies including teenage pregnancy, promotes sex and relationships education, brings about change in prejudice, stigma and discrimination, and general awareness-raising work" (Thompson et al. 2008, 319). Health promotion scholarship is said to be "emblematic of wider contemporary social and cultural changes - changes which are characteristic of the acceleration of processes associated with later 
modernity (Giddens 1990; 1992), the rise of the risk society (Beck 1992a; 1992b), and the growing preoccupation with the body, lifestyle, and consumer culture” (Burrows et al. 1995). In definitions of health under late modernity, symptoms, signs and illness are often positioned as predictive risk factors (Armstrong, 1995). With this conception of health, risky sexual behaviours, such as number of sexual partners or low levels of contraceptive use (including condoms), are seen to have serious health consequences, including prevalence of HIV or STI infection (Allen et al. 2003). As Pratt $(2000,4)$ discusses, "although it is clear that any definition of sexual health means more than just simply the absence of sexual function or disease, no definition can ignore the fact that the pandemic of all forms of sexually transmitted diseases, including HIV and hepatitis B virus (HBV) infection, precludes many people throughout the world from enjoying sexual health”. With this very brief discussion of sexual health promotion and risk, we will now discuss how these themes converge with subject headings in LCSH and RVM.

LCSH and RVM converge with discourses due to literary warrant. There are 782 records in the Library of Congress's OPAC that use the subject heading “Sexual Health”. While theoretically the sexual health subject heading can be built with topical, form, geographical, and chronological subdivisions, currently geographical subdivisions (e.g., Africa, Brazil, Chile) and form subdivisions (e.g., Audiovisual aids, Dictionaries, Government Policy) dominate. There are records in the Library of Congress OPAC that reflect the WHO's understanding of sexual health, but there are no corresponding subject headings to access this material. For instance, The psychology of sexual health (Miller 2002) is currently indexed under "Sex (Psychology)" and "Sexual Health", and not under "Sexual Health-Psychological aspects", which could be a possible subject heading. Using the OPAC of the Bibiliothèque de l’Universisté Laval, for which RVM is built, we can observe the same situation. For the subject heading "hygiène sexuelle", there are 72 records, and no subdivisions have been added to reflect, for example, the psychological consequences of sexual health. Similarly, Reproductive \& sexual health rights in Cameroon, Egypt, Ghana, Malawi, and Rwanda: An advocacy and communications approach (Musa and Ndomo 2007) is currently under "Reproductive Rights - Africa” and "Sexual Rights - Africa", which obscures the role of sexual health altogether. To access the 10 themes associated with sexual health above, users must employ a combination of subject headings, such as "Sexual Health" and "Sexually Transmitted Diseases". How these subject headings are applied to records is somewhat unclear. For instance, there are 132 records in the Library of Congress's OPAC that have the phrase “sexual health” in their main title and 101 of these 132 records have associated subject headings. Looking closer, we found that only 45 (less than half) of the 101 records were indexed with the subject heading "Sexual Health". The other 56 records are indexed with a myriad of subject headings, the most frequent of which were "Reproductive Health" (11), "Sex" (11), "Sexually Transmitted Diseases" (10), “Teenagers" (15), and "Sexual Behavior" (10). Again, we can see the same pattern for RVM. While subject headings have the potential to offer greater access to resources within a library OPAC, in the case of sexual health the inconsistent application of the "Sexual Health" subject heading and the limited number of subdivisions applied to this subject heading, limit access to relevant materials.

\subsection{Resistance}

In framing their understanding of resistance, Bowker and Star (1999) draw on Latour's (1987) statement that reality is that which resists. While the limitations of LCSH have been detailed since the late 1960s, the reality is that this system continues to dominate and that efforts 
are being taken by the Library of Congress to ensure the continued usefulness of LCSH. As Knowlton $(2005,124)$ discusses, LCSH’s “dominance within the libraries in the United States, coupled with the export of OPAC technology from American companies to libraries around the world, has led to the adoption of $L C S H$ in libraries around the world”. In this section we discuss some of the potential future changes in LCSH and how this might impact the representation of sexual health.

It is important to recognize that the Library of Congress is not considered a national library and receives no funding for bibliographic services which "compromises its continuing ability to carry out functions depended upon by many of the nation's libraries” (Library of Congress 2008, 6). Its willingness to continue work in this direction has "greatly benefited libraries in the United States and throughout the world" (Library of Congress 2008, 6). In order to ensure the continued usefulness of LCSH, the Library of Congress $(2008,32)$ encourages "the enhancement of library systems to provide the capability to link to appropriate user-added data available via the Internet (e.g., Amazon.com, LibraryThing, Wikipedia). The potential benefits of user-generated content as an alternative or enhancement to controlled vocabulary have been cited by several authors (Mathes 2004; Kipp 2008). For instance, in comparing LCSH to LibraryThing, Mendes et al. (2009, 39) found that "for every new book a user discovers using LCSH headings they will discover four books using LTFL [LibraryThing for Libraries]", but that the ability of these tags to return relevant resources must still be assessed. Rolla $(2009,182)$ similarly compared LibraryThing to LCSH and found that "while user tags can enhance subject access to library collections, they cannot replace the valuable functions of controlled vocabulary like LCSH”.

In order to consider the potential future representation of sexual health through the collaboration of LCSH with user-added data, we compared subject headings for sexual health in the Library of Congress to one source of user-general content, LibraryThing (see appendix I, figure 2). Five out of ten of the top themes identified in figure 1 were found as tags on LibraryThing (sex, HIV/AIDs, sexuality, sexual education, and pregnancy) and five were not available (risk, knowledge or information, STIs or STDs, reproduction and condoms). Some of the themes mentioned in WHO's definition of sexual health were available on LibraryThing (e.g., psychology), but most were also unavailable (e.g., emotional, mental, and social wellbeing, pleasure, violence, and sexual rights). Other tags are available through LibraryThing that are not mentioned by WHO or found as the most frequently occurring themes listed above (e.g., self-help, plays, feminist, glbt). How this application could enhance LCSH at a national level has yet to be determined. From this brief investigation one could conjecture, like Rolla (2009), that user tags could enhance but not replace LCSH.

\section{Discussion}

In this paper we have investigated the representation of sexual health in LOC and RVM subject headings through three of Bowker and Star's (1999) infrastructural inversion techniques, practical politics, convergence, and resistance. The effects of these strategies are often impossible to differentiate as, for example, the design features of the system (practical politics) impact topical representation of the subject headings and how these headings undergird social worlds (convergence). In combination, however, Bowker and Star's (1999) strategies enable us to make visible the subtle features of our knowledge organization systems. As knowledge organization systems, such as subject heading lists, become more embedded "they risk getting 
black boxed and thence made both potent and invisible” (Bowker and Star 1999, 325). Making transparent the voices and practices of designers can help these knowledge organization systems to "retain maximum political flexibility" (Bowker and Star 1999, 325).

Investigation of practical politics, or the design features of LCSH and RVM, reveals that LCSH and RVM do not represent a holistic understanding of sexual health as defined by the WHO (2002). Even though RVM represents the topic of sexual health in a more holistic way, it is still incomplete. Investigation of convergence, or the mutual constitution of social worlds and information infrastructures, reveals that the inconsistent application of the LCSH "Sexual Health" and the limited number of subdivisions applied to this heading limit access to relevant resources. A significant number of sexual health resources in the Library of Congress OPAC were not connected to the "Sexual Health" subject heading. Sexual health resources were instead linked to such subject headings as "Sex", which has no associative reference to "Sexual Health" for Library of Congress cataloguers. And investigation of resistance, or the reality that LCSH creates through its continued presence, reveals that in spite of all of its flaws LCSH represents an extensive and rich vocabulary that can be enhanced but not replaced by user-generated content.

Investigations of knowledge organization systems through Bowker and Star's (1999) infrastructural inversion techniques represent a non-standard way of reading these systems. We hope to show that these techniques can help to make our systems more transparent. Other nonstandard methods for investigating the design principles of our information organization systems are also available. For instance, Roth's (2009) investigation of scientists' “classification” practices through ethnographic research reveals the tacit process through which scientists' identify an unknown specimen. Roth's $(2009,612)$ analysis reveals that "monster categories and self depreciation presumably are expressions of scientists' understanding of the uncertainty involved in making certain classifications”. A similar investigation of how LCSH and RVM designers' deal with "monster categories" could also help to reveal the tacit knowledge that is buried within our information systems, which may help to ensure the continued flexibility and adaptability of these systems.

\section{References}

Allen, Denise Roth, James W. Carey, Chomnad Manopaiboon, Richard A. Jenkins, Wat Uthaivoravit, Peter H. Kilmarx and Frits van Griensven. 2003. Sexual health risks among young thai women: Implications for HIV/STD prevention and contraception. AIDS \& Behavior, 7: 9-21.

Armstrong, David. 1995. The rise of surveillance medicine. Sociology of Health \& Illness 17: 393-404.

Beck, Ulrich. 1992a. Risk society: towards a new modernity. London: Sage.

Beck, Ulrich. 1992b. From industrial society to the risk society: questions of survival, social structure and ecological enlightenment. Theory, Culture \& Society 9: 97-123.

Bélair, Jo-Anne, Bélanger, Sylvie, Dolbec, Denise and Hudon, Michèle. 2009. Guide pratique du Répertoire des vedettes-matière de l'Université Laval. Montreal: Asted.

Bialek, Ron, Grace L. Duffy and John W. Moran. 2009. The public health quality improvement handbook. Milwaukee, Wis.: ASQ Quality Press.

Bowker, Geoffrey C. and Susan Leigh Star. 1999. Sorting things out: classification and its consequences. Cambridge, Mass.: MIT Press. 
Burrows, Roger, Sarah Nettleton and Robin Bunton. 1995. Sociology and health promotion: health, risk and consumption under late modernity. In The sociology of health promotion: critical analyses of consumption, lifestyle and risk, edited by R. Bunton, S. Nettleton, and R. Burrows, 1-12. New York: Routledge.

Chan, D. J., and B. Donovan, B. 2005. What's in a word? Case definitions in sexual health medicine. International Journal of STD \& AIDS 16n2: 91-94.

Chan, Lois Mai. 2005. Library of Congress subject headings: principles and application. 4th ed. Westport, Conn.: Libraries Unlimited.

Dolbec, Denise. 2006. Le répertoire de vedettes-matière : outil du XXIe siècle. Documentation et bibliothèques, 52n2: 99-108.

Edwards, Weston M. and Eli Coleman. 2004. Defining sexual health: a descriptive overview. Archives of Sexual Behavior 33: 189-195.

Feinberg, Melanie. 2007. Hidden bias to responsible bias: an approach to information systems based on Haraway's situated knowledges. Information Research 12n4. Available: http://informationr.net/ir/12-4/colis/colis07.html.

Fischer, Karen S. 2005. Critical views of LCSH, 1990-2001: the third bibliographic essay. Cataloging \& Classification Quarterly 41n1: 63-109.

Giddens, Anthony. 1991. Modernity and self-identity: self and society in the late modern age. Cambridge: Polity.

Giddens, Anthony. 1992. The transformation of intimacy. Cambridge: Polity.

Hjørland, Birger, and Hanne Albrechtsen. 1995. Toward a new horizon in information science: domain analysis. Journal of the American Society for Information Science 56: 400-425.

Jordan, Carol E. 2009. Advancing the study of violence against women: evolving research agendas into science. Violence Against Women 15: 393-419.

Kipp, Margaret E. I. 2008. Searching with tags. Do tags help users find things? In Culture and Identity in Knowledge Organization: Proceedings from the Tenth International ISKO Conference, 5-8 August 2008 Montréal, Canada. Würzburg: Ergon Verlag.

Knowlton, Steven A. 2005. Three decades since prejudices and antipathies: a study of changes in the library of congress subject headings. Cataloging \& Classification Quarterly 40: 123145.

Latour, Bruno. 1987. Science in action: how to follow scientists and engineers through society. Cambridge, Mass.: Harvard University Press.

Library of Congress. 2008. On the record: report of the Library of Congress working group on the future of bibliographic control. Available: http://www.loc.gov/bibliographicfuture/news/lcwg-ontherecord-jan08-final.pdf.

Mai, Jens-Erik. 2010. Classification in a social world: bias and trust. Journal of Documentation 66: 627-642.

Mathes, Adam. 2004. Folksonomies: cooperative classification and communication through shared metadata. Available: http://www.adammathes.com/academic/computer-mediatedcommunication/folksonomies.html.

McDowell, Ian. 2006. Measuring health: a guide to rating scales and questionnaires. $3^{\text {rd }}$ ed. New York: Oxford University Press.

McTavish, Jill, Diane Neal and C. Nadine Wathen. Forthcoming. Is what you see what you get? medical subject headings and their organizing work in the violence against women research literature. Knowledge Organization. 
Mendes, Luis H., Jennie Quiñonez-Skinner and Danielle Skaggs. 2008. Subjecting the catalog to tagging. Library Hi Tech 27n1: 30-41.

Miller, David and John Green. 2002. The psychology of sexual health. Oxford: Blackwell Science.

Musa, Roselynn and Atienno Ndomo. 2007. Reproductive and sexual health rights in Cameroon, Egypt, Ghana, Malawi, and Rwanda: an advocacy and communications approach. Nairobi: FEMNET.

Olson, Hope A. 2000. Difference, culture and change: the untapped potential of LCSH. Cataloging \& Classification Quarterly 29: 53-71.

Olson, Hope. A. and Dennis B. Ward. 1997. Ghettoes and diaspora in classification: communicating across the limits. In Communication and Information in Context: Society, Technology, and the Professions: Proceedings from the annual conference of the Canadian Association for Information Science, June 8-10, St. John's, Canada. Available: http://www.cais-acsi.ca/proceedings/1997/Olson_1997.pdf

Pratt, Robert. 2000. Sexual health and disease: an international perspective. In Sexual health: foundations for practice, edited by Heather Wilson and Sue McAndrew, 1-8. Edinburgh: Harcourt.

Répertoire de vedettes-matières. 2011. About us. Available: https://rvmweb.bibl.ulaval.ca/en/apropos

Rolla, Peter J. 2009. User tags versus subject headings. Library Resources \& Technical Services 53: 174-184.

Roth, Wolff-Michael. 2005. Making classifications (a) work: ordering practices in science. Social Studies of Science 35: 581-621.

Shorter Oxford English dictionary. 2007. $6^{\text {th }}$ ed. Oxford: Oxford University Press.

Thompson, Kate, Karen Casson, Paul Fleming, Frank Dobbs, Kader Parahoo and Janice Armstrong .2008. Sexual health promotion in primary care: activities and views of general practitioners and practice nurses. Primary Health Care Research \& Development, 9: 319-330.

WHO. 1986. Ottawa charter for health promotion. Ottawa: World Health Organization.

WHO. 1987. Concepts of sexual health. Report on a working group 5-7 May 1987, Copenhagen. Copenhagen: World Health Organization.

WHO. 2002. Defining sexual health. Report of a technical consultation on sexual health 2831January 2002, Geneva. Geneva: World Health Organization. 
Jill McTavish and Alexandre Fortier. 2011. A domain-analytic perspective on sexual health in LCSH and RVM. In Smiraglia, Richard P., ed. Proceedings from North American Symposium on Knowledge Organization, Vol. 3. Toronto, Canada, pp. 83-93.

\section{Appendix I}

Figure 1. Flowchart showing the number of citations retrieved by individual searches for the years of $1980,1990,2000$, and 2010, the number of citations subjected to word frequency analysis, and the number of citations referencing the ten most frequently occurring themes associated with sexual health.

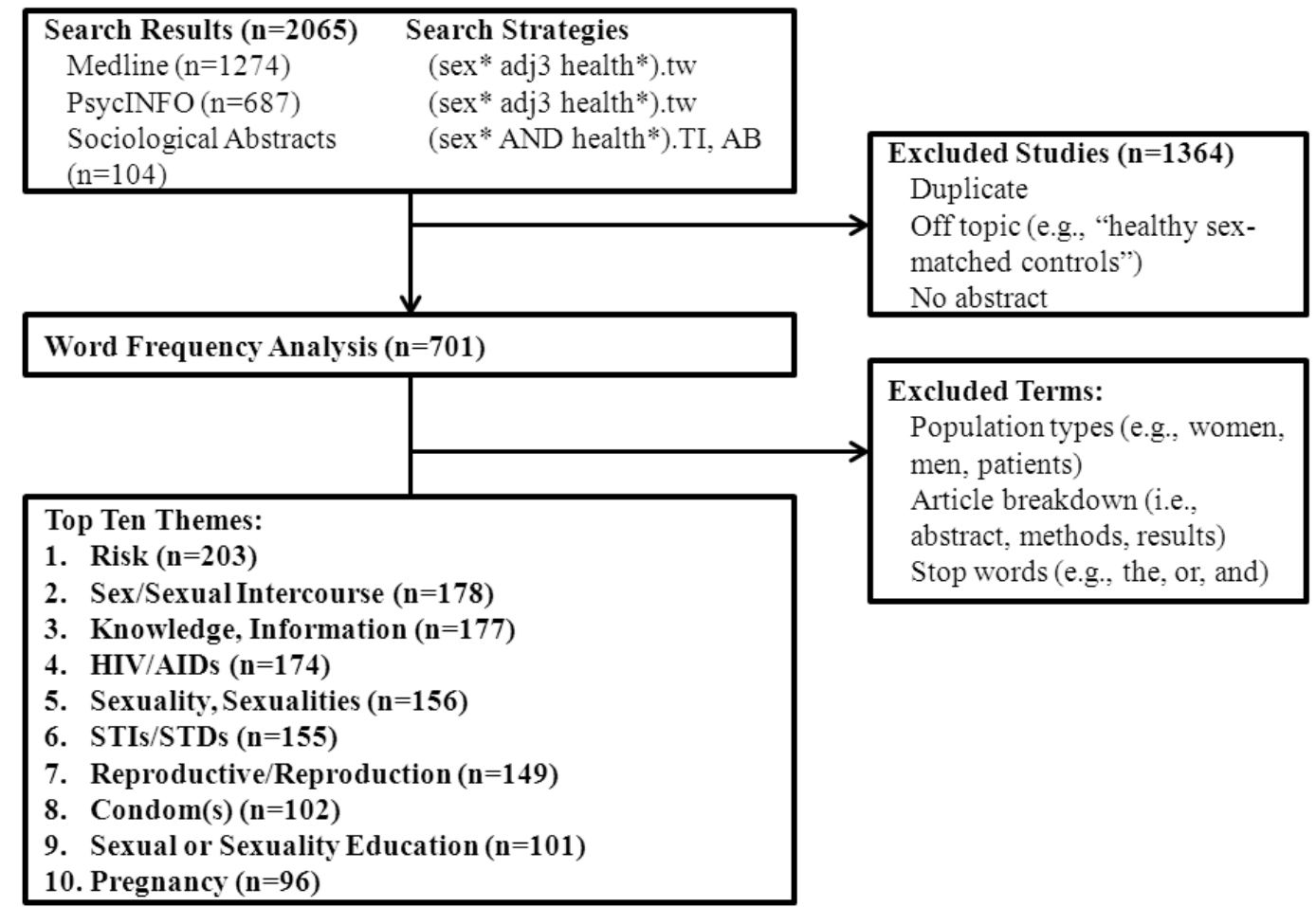

Figure 2. Tags and related LCSH subjects for sexual health found in LibraryThing in May, 2011 (http://www.librarything.com/tag/sexual+health).

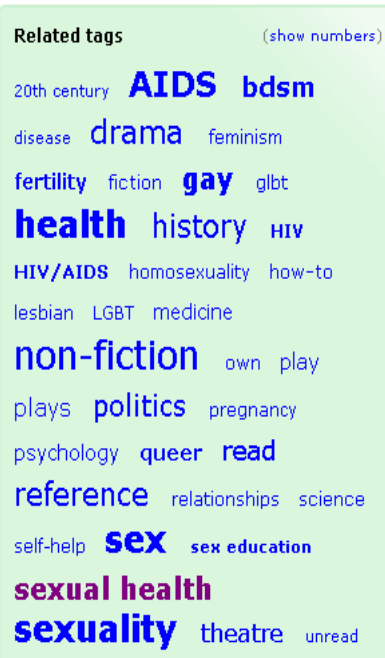

